\title{
PHYSICAL REVIEW A
}

\section{ATOMIC, MOLECULAR, AND OPTICAL PHYSICS}

\section{RAPID COMMUNICATIONS}

The Rapid Communications section is intended for the accelerated publication of important new results. Since manuscripts submitted to this section are given priority treatment both in the editorial office and in production, authors should explain in their submittal letter why the work justifies this special handling. A Rapid Communication should be no longer than 4 printed pages and must be accompanied by an abstract. Page proofs are sent to authors.

\section{Anomalous asymmetry splittings in a molecule with internal rotation in the presence of classical chaos}

\author{
Juan Ortigoso \\ Instituto de Estructura de la Materia, CSIC, Serrano 119, 28006 Madrid, Spain
}

(Received 12 March 1996)

\begin{abstract}
Rotation-torsion energy levels of acetaldehyde calculated from a spectroscopic Hamiltonian obtained from the fit of high-resolution microwave and far-infrared spectra, exhibit anomalously large splittings for some asymmetry doublets. It is shown that these anomalies are caused by chaos-enhanced quantum dynamical tunneling between classically disconnected regions of the phase space. A major consequence of this phenomenon is the magnification of intramolecular energy-transfer processes. [S1050-2947(96)50310-0]
\end{abstract}

PACS number(s): 03.65. $-\mathrm{w}, 05.45 .+\mathrm{b}, 34.50 . \mathrm{Ez}$

Acetaldehyde $\left(\mathrm{CH}_{3} \mathrm{CHO}\right)$ is a slightly asymmetric top molecule containing a quantum gyroscope (the $\mathrm{CH}_{3}$ group) which can internally rotate with respect to the $\mathrm{CHO}$ frame. This Rapid Communication shows that chaotic coupling between the internal rotation of the $\mathrm{CH}_{3}$ group (torsional vibration) and the overall molecular rotation gives rise to enhanced dynamical tunneling [1] between disconnected regions of the classical phase space causing anomalous splittings for some asymmetry doublets. These effects are relevant to current spectroscopic investigations of the role of internal rotation on intramolecular vibrational relaxation (IVR) processes. They are also relevant to the study of the signature of classical chaos in a pure quantum-mechanical process like tunneling.

Rotation-torsion energy levels of acetaldehyde can be classified using the Longuet-Higgins permutation-inversion group $G_{6}$ [2]. Accordingly, the symmetry of the energy levels will be $A$ or $E$. $A$-symmetry energy levels come in $\left(A_{1}, A_{2}\right)$ nearly degenerate pairs called asymmetry doublets or $K$ splittings ( $K$ is the absolute value of the projection of the total angular momentum along a body-fixed axis), and their pattern resembles, in general, that of a pseudorigid asymmetric top. However, $E$ levels are degenerate and they normally exhibit a qualitatively different pattern.

Numerous studies trying to assess the influence of internal rotation on IVR have been published in the recent past [3-5]. Usually torsion is the lowest vibration in molecules in which it is present; therefore, at moderate to high energies, a sig- nificantly higher density of states than in analogous molecules with no torsional vibration is found. This fact has been proposed to cause an enhancement of IVR by means of torsion-assisted vibrational couplings. Recently Lees [5] has shown that splittings of asymmetry doublets in excited vibrational states of methanol, another prototype molecule for internal rotation studies, are very sensititive to intermode coupling. This sensitivity gives rise to anomalous splittings that can be exploited, using Lees's wording, as a useful tool for the study of torsion-mediated coupling and intramolecular energy-transfer channels in methanol. In this Rapid Communication, a different mechanism that also produces anomalous $K$ splittings in molecules containing an internal rotor is shown to exist.

On the other hand, the relation between chaos and tunneling is not yet clear, although some progress has been made in the recent past. Latka et al. [6] have studied a chaos-assisted avoided crossing phenomenon for the driven pendulum, and Tomsovic and Ullmo [7] have shown how chaos can assist tunneling in a system of two coupled quartic oscillators. In these systems the two components of a regular doublet are split by dynamical tunneling. When an external parameter is changed, a third state, which happens to be chaotic, enters into play. This state can interact with only one of the components of the regular doublet changing the splitting pattern. This phenomenon is caused because "chaotic" eigenvalues of the driven pendulum or the coupled quartic oscillator do not have a symmetric partner in the phase space and therefore only the state with the right symmetry is affected by the 
(a) $E=300 \mathrm{~cm}^{-1}$

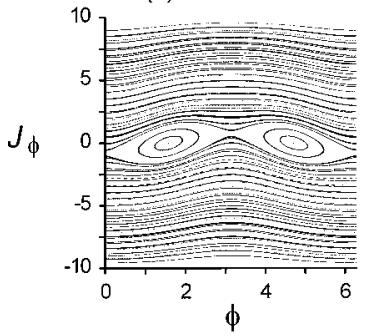

(c) $E=495 \mathrm{~cm}^{-1}$

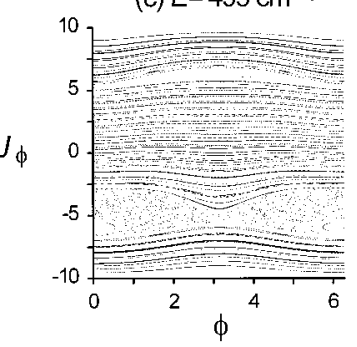

(b) $E=434 \mathrm{~cm}^{-1}$

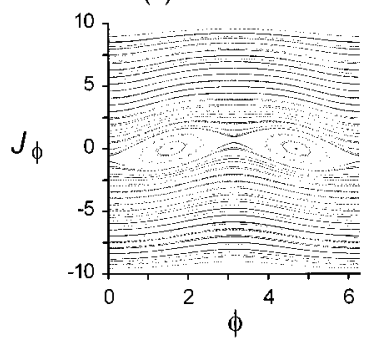

(d) $E=707 \mathrm{~cm}^{-1}$

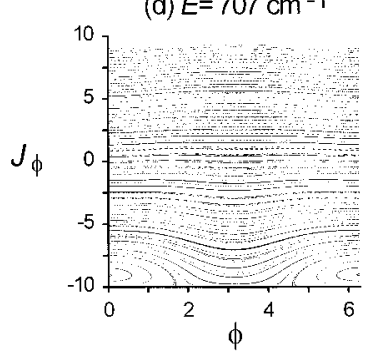

FIG. 1. Composite Poincaré surfaces of section for acetaldehyde at $J=10$. Each surface corresponds to a different total (torsional plus rotational) energy. The surfaces are defined by $p_{\alpha}>0$, $\alpha=2 \pi / 3$, and represent the variation of the projection of the angular momentum along the $a$ axis $\left(J_{\phi}=k\right)$ of the $\rho$-axis system with respect to its conjugate Euler angle $(\phi)$ expressed in radians. (c), which corresponds to an energy similar to the top of the barrier to internal rotation, shows two chaotic regions.

presence of the chaotic state. A different case has been discussed by Roncaglia et al. [8]. These authors related the tunneling rate between stable islands of the classical phase space for the kicked Harper model to the size of the chaotic region separating the islands. A relation between the size of the tunneling splitting and the overlap of the Husimi representation of the two eigenvectors involved in a doublet with the chaotic zones of the classical phase space was proposed previously by Utermann et al. [9] for the periodically driven quartic double well.

The Hamiltonian used to model rotation-torsion energy levels of acetaldehyde, written in the convenient $\rho$-axis system [2], which can be obtained from the principal-axis system by a rotation, is

$$
\begin{aligned}
H= & F\left(p_{\alpha}-\rho J_{z}\right)^{2}+\left(V_{3} / 2\right)(1-\cos 3 \alpha)+A J_{a}^{2}+B J_{b}^{2}+C J_{c}^{2} \\
& +D_{a b}\left(J_{a} J_{b}+J_{b} J_{a}\right)
\end{aligned}
$$

where $J_{a}, J_{b}$, and $J_{c}$ are the components of the total angular momentum in the $\rho$-axis system, $\alpha$ is the torsional angle, and $p_{\alpha}$ is its conjugate momentum. The parameters $A, B, C$, and $D_{a b}$ are rotational constants, $F$ is the rotational constant associated with internal rotation, $\rho$ is a parameter involving moments of inertia of the $\mathrm{CH}_{3}$ top and the $\mathrm{CHO}$ frame in the molecule, and $V_{3}$ is the height of the torsional barrier hindering the internal rotation. Values for these spectroscopic constants were obtained by Kleiner and co-workers [10] from a fit of microwave and far-infrared transitions on acetaldehyde. Therefore, these parameters contain the influence of the small-amplitude vibrations through high-order terms in the perturbation expansions commonly used by spectros- (a) $v_{f}=1$

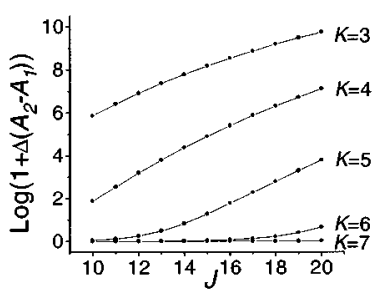

(c) $v_{t}=3$

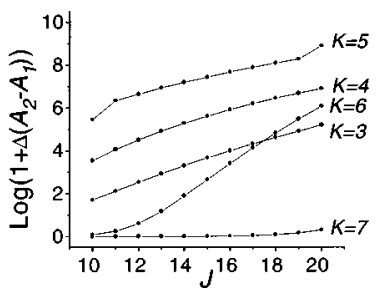

(b) $v_{t}=2$

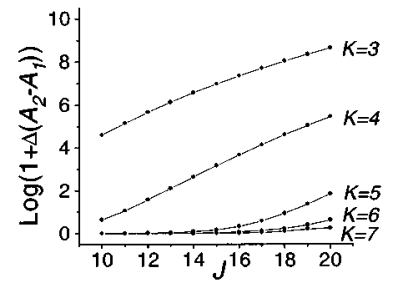

(d) $v_{f}=4$

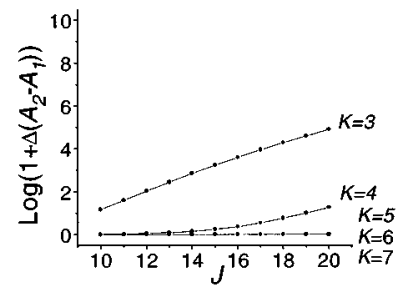

FIG. 2. $\log _{10}$ of $\left[1+\Delta\left(A_{2}-A_{1}\right)\right]$ for selected asymmetry doublets of acetaldehyde as a function of the total angular momentum $J$. $\Delta\left(A_{2}-A_{1}\right)$ represents the absolute value (in $\mathrm{MHz}$ ) of the $K$ splitting of an $A_{1}, A_{2}$ pair. The most anomalous splittings belong to the curves labeled $K=5$ and $K=4$ for $v_{t}=3$. For high $J$ the splittings for levels with $v_{t}=3$ and $K=6$ are also anomalous.

copists. The complete Kleiner and co-workers Hamiltonian includes many more terms needed to fit the spectrum to experimental measurement precision (ranging from $4 \mathrm{KHz}$ for the more precise microwave measurements to $15 \mathrm{MHz}$ for infrared transitions), but the features relevant to the present paper are well described by the truncated effective Hamiltonian given by Eq. (1).

Acetaldehyde is more asymmetrical than methanol and a complete local breakdown of the $k$ quantum number (signed $K$ ) is feasible because of strong coupling between torsion and rotation. In Fig. 1, Poincaré surfaces of section computed from a classical Hamiltonian [obtained from Eq. (1) by replacing the angular-momentum components by their classical limits: $J_{a}=J \cos \theta ; J_{b}=-J \sin \theta \cos \phi ; J_{c}=J \sin \theta \sin \phi$; $\theta$ and $\phi$ being spherical polar angles defining the direction of the angular-momentum vector [11]] show that at unusually low energies (about $450 \mathrm{~cm}^{-1}$ for $J=10$ ) chaos develops, giving rise to a mixed phase space, where chaotic and regular regions coexist. This energy range corresponds to the top of the barrier to internal rotation (the barrier height, $V_{3}$, is 408 $\left.\mathrm{cm}^{-1}[10]\right)$. It is precisely in this regime that the time scale of the torsional tunneling is comparable to the overall rotation and the adiabatic separation between both motions breaks down. At higher energies and the same total angular momentum the molecule is in a different limiting case in which the $\mathrm{CH}_{3}$ group rotates almost freely and the interaction with the overall rotation is greatly reduced. As a consequence, chaotic regions are not visible anymore in the Poincaré surfaces of section and the projection of the total angular momentum along one of the body-fixed axes is again approximately conserved, as can be seen in Fig. 1(d).

The relationship between the invariant structures of classical mechanics and quantum-mechanical eigenvectors can be understood by inspection of generalized Husimi distributions [12]. These distributions give a probability density 
which is a measurement of the overlap of the eigenvectors with coherent states centered at the classical points of interest. Since our system has two degrees of freedom, surfaces of section are useful to visualize the Husimi distributions and they also provide an easier comparison with the Poincare surfaces of section [13]. In brief, quantum rotational surfaces of section are contour plots of a probability density $\rho_{E}(\Omega)=\left|\left\langle\Omega \mid \Psi_{E}\right\rangle\right|^{2}$ ( $\Omega$ being the coherent state) evaluated on the $(\phi, k)$ plane defined by the conditions $\langle\Omega|H| \Omega\rangle=E$ ( $E$ is the eigenvalue corresponding to the eigenvector $\Psi_{E}$ ), $p_{\alpha}>0$, and $\alpha$ fixed to a constant.

Coherent states for the present problem have been chosen as direct products of SU(2) coherent states (extremal states for a rigid asymmetric top), multiplied by harmonicoscillator coherent states with periodic boundary conditions, which are appropriate for torsional vibrations. The SU(2) coherent states are parametrized by two Euler angles and can be expanded in terms of the eigenstates of $J^{2}$ and $J_{a}$ [13],

$$
\begin{aligned}
|\theta \phi\rangle= & \sum_{k=-J}^{J} \sin ^{J+k}(\theta / 2) \cos ^{J-k}(\theta / 2) \\
& \times\left(\begin{array}{c}
2 J \\
J+k
\end{array}\right) e^{-i(J+k) \phi}|J, k\rangle .
\end{aligned}
$$

Coherent states for the torsional degree of freedom are chosen as Fourier expansions $\left(\sum_{n} c_{n} e^{i n \alpha}\right)$ of a Gaussian wave packet

$$
\Phi_{p_{\alpha}, \alpha_{0}}(\alpha)=\frac{1}{\left(2 \pi \sigma^{2}\right)^{(1 / 4)}} \exp \left(\frac{-\left(\alpha-\alpha_{0}\right)^{2}}{4 \sigma^{2}}+i p_{\alpha} \alpha\right) .
$$

A $\sigma^{2}$ value of 0.07 assures faithful phase-space representations according to Ref. [6]. The coefficients of the Fourier series are given by

$$
\begin{aligned}
c_{n}= & \left(\frac{\pi \sigma^{2}}{2}\right)^{(1 / 4)} \exp \left[\sigma^{2}\left(2 n p_{\alpha}-n^{2}-p_{\alpha}^{2}\right)+i \alpha_{0}\left(p_{\alpha}-n\right)\right] \\
& \times\left[\omega\left(\frac{\pi}{2 \sigma}+i \sigma\left(p_{\alpha}-n\right)\right)+\omega\left(\frac{\pi}{2 \sigma}-i \sigma\left(p_{\alpha}-n\right)\right)\right],
\end{aligned}
$$

where $\omega$ is the error function.

The eigenvectors of $H$ in the basis set $|m\rangle|J, k\rangle$, where $|m\rangle$ are free rotor wave functions $\left(e^{i m \alpha}\right)$, are highly mixed, with contributions from many basis functions. Nonetheless, in most cases one of the rotational basis functions gives the biggest contribution and a nominal $k$ label can be ascribed to the corresponding eigenvalues. In some cases a redefinition of the axis system using rotational energy surfaces (RES) is needed to get unambiguous $k$ quantum numbers [14]. The RES concept is an important tool for the study of complex spectral and dynamical phenomena, but when the adiabatic separation between the overall rotation and other degrees of freedom breaks down, as happens in the present problem, a single RES treatment is not useful. However, a multiple surface treatment could still be interesting [15]. Eigenvectors of $H$ can be expressed alternatively as linear combinations of $\left|v_{t}\right\rangle|J, k\rangle$ basis functions, where $\left|v_{t}\right\rangle$ are eigenvectors of the torsion- $K$-rotation part of $H$. Use of this basis set allows us to impose in most cases an approximate torsional quantum (a) $E=300.656, v=1, K=5$

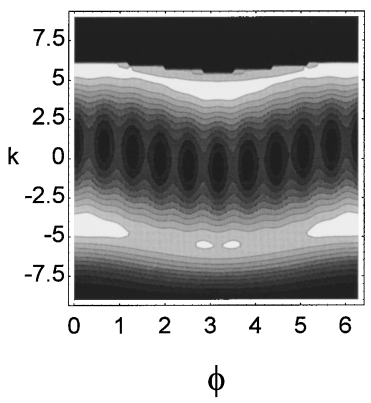

(c) $E=495.138, v=3, K=5$

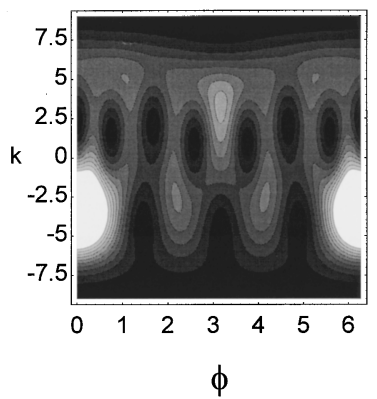

(b) $\mathrm{E}=434.247, v=2, \mathrm{~K}=5$

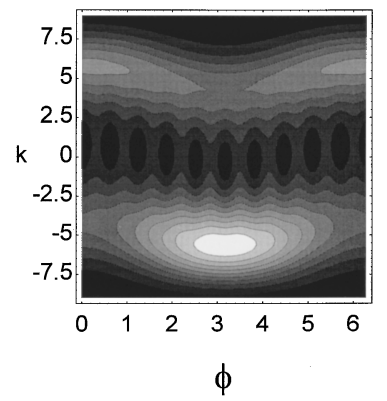

(d) $E=707.249, v=4, K=5$

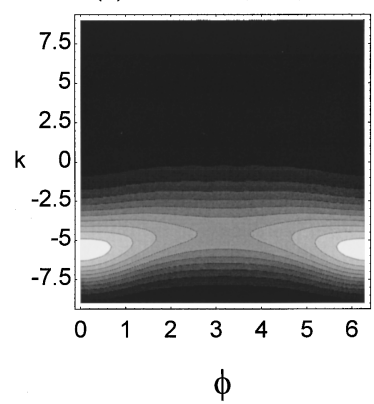

FIG. 3. Quantum rotational surfaces of section in the $(\phi, k)$ plane (with $\phi$ expressed in radians) for four different eigenvectors of acetaldehyde at $J=10$. The surfaces are defined by $p_{\alpha}>0$, and $\alpha=2 \pi / 3$. Each surface is labeled by the corresponding eigenvalue (in $\mathrm{cm}^{-1}$ ) and the nominal quantum numbers $v_{t}$ and $K$ (see text). The gray scale is such that darker contours represent smaller densities.

number (given by the $\left|v_{t}\right\rangle$ function with the biggest contribution) to torsion-rotation eigenvectors.

A plot of the tunneling splittings between the two components of a number of asymmetry doublets of acetaldehyde, calculated from the spectroscopic Hamiltonian given by Eq. (1) is shown in Fig. 2. The normal behavior of the splittings is illustrated by Figs. 2(a), (b), and (d): they decrease with the increase of $K$, and with the increase of the torsional quantum number $v_{t}$. This is the expected behavior for wellbehaved molecules with an internal rotor, i.e., molecules for which the coupling between torsion and rotation is not strong. This trend is broken for levels with $K=4$ or 5 , and $v_{t}=3$ for every $J$, as can be seen in Fig. 2(c). In these cases the splittings are much larger than expected. For high $J$ values the splittings of asymmetry doublets with $v_{t}=3$ and $K=6$ are also anomalously large.

Quantum surfaces of section for four eigenvectors with $J=10$ and $K=5$ corresponding to different torsional states are shown in Fig. 3. Distinctive characteristics can be observed in the quantum surface of the eigenvector corresponding to an $\left(A_{1}, A_{2}\right)$ pair with anomalous splitting $\left(E=495.138 \mathrm{~cm}^{-1}, v_{t}=3\right)$. This surface presents well localized maxima inside the classically chaotic region [see Fig. $1(\mathrm{c})]$, and contours with smaller but still significant density delocalized over large regions of the surface, connecting the $+K$ and $-K$ zones. This behavior is found in all the quantum surfaces of section of eigenvectors involved in anomalous splittings, but not in those with regular splittings. The delocalization seems to occur only when the maximum (or maxima) of the quantum density lies on a chaotic region of 
the Poincare surface of section. In this sense, the size of the tunneling splitting can be related to the overlap of the $\mathrm{Hu}$ simi representations of the pair of eigenvectors involved in the doublets with the chaotic zones of the classical phase space, as was proposed by Utermann et al. [9]. This relation is also in agreement with the anomalous splittings found for pairs with $K=6$ for high $J$ [see Fig. 2(c)] since the relative size of the chaotic regions increases with $J$. There are, however, important differences between the phase-space structures and Husimi distributions for the rotation-torsion problem in acetaldehyde and for the periodically driven quartic double well that can be relevant to understand the connection between chaos and tunneling. In the double well, the tunneling rate changes when the two quantizing tori related to the states involved in a particular doublet resolve in a common chaotic region of the classical phase space, and contributions to the tunneling splitting from paths which pass through the chaotic layer become dominant. In the case of acetaldehyde the eigenstates involved in the anomalous splittings originate in two different chaotic regions where many caustics exist, from each of which tunneling can occur as suggested by Takatsuka and Ushiyama [16] for the Hamilton-Jacobi equation.

Eigenstates whose Husimi distributions present maxima in regular regions can also show significant density on both the positive- and the negative- $K$ regions, like those for $v_{t}=1$ and 2 in Fig. 3, since these wave functions are, using Heller's nomenclature [17], dirty mixings of tori. However, in these cases the region in between has zero density and the $\left(A_{1}, A_{2}\right)$ pairs of rotation-torsion states are not noticeably split. Nonstationary wave packets corresponding to linear combinations of two dirty eigenvectors will be well localized in, for example, the positive- $K$ region of the phase space and they will tunnel very slowly to the negative- $K$ region. In the presence of chaos, dynamical tunneling is much enhanced and the time for an initially localized wave packet to tunnel back and forth between the $+K$ and $-K$ regions will be much shorter. For example, a wave packet formed by a linear combination of the two components of the doublet labeled by $v_{3}=3$ and $K=5$ will tunnel in about $4 \mathrm{~ns}$. On the other hand, a wave packet created from the two components of the doublet with $v_{t}=2$ and $K=5$ needs about $0.6 \mathrm{~ms}$. Consequently, state-to-state rotational energy transfer caused by dynamical tunneling becomes more important in the presence of classical chaos.

Degradation of quantum numbers by dynamical tunneling has recently been proposed by Heller [17] as a mechanism for enhanced IVR in molecules excited in energy regions with a high density of states but no chaos. States in this regime normally have multiple quanta of excitation in the small-amplitude vibrations. In this case the resonance scheme is more complex, since more than two degrees of freedom must be considered explicitly. However, the strong coupling between the two degrees of freedom in the torsionrotation subsystem that gives rise to augmented dynamical tunneling and enhanced rotational energy transfer in the lowdensity-of-states regime remains. In other words, the degradation of $k$ will occur also at energies where IVR can take place, increasing vibration-rotation couplings. Therefore, the present work suggests that restricted chaos can also play an indirect role on IVR.

Finally, when the limiting case for which the torsion can be considered as independent from the overall rotation is reached, tunneling is completely suppressed. This behavior is illustrated by the quantum surface of section for the eigenstate labeled by $v_{t}=4$ and $K=5$ in Fig. 3(d), which has significant density only in the negative- $K$ zone.

The recent interest in the study of the interplay between chaos and tunneling is producing new concepts that need experimental confirmation. The relation found in the present Rapid Communication between classical chaos and anomalous splittings for some asymmetry doublets of acetaldehyde in an easily accessible energy region suggests that highresolution molecular spectroscopy with its highly developed experimental techniques can offer a unique tool for testing this kind of theories.

This work was supported in part by the Division of Chemical Sciences, Office of Basic Energy Sciences, Office of Energy Research, U.S. Department of Energy, and by the Spanish DGICYT (Research Project No. PB93-0138). The author is indebted to Jon Hougen and Isabelle Kleiner for numerous discussions and for permission to use some of their computer programs, and to W. P. Reinhardt for suggesting the use of quantum surfaces of section to study spectral phenomena.
[1] M. J. Davis and E. J. Heller, J. Chem. Phys. 75, 246 (1981).

[2] J. T. Hougen, I. Kleiner, and M. Godefroid, J. Mol. Spectrosc. 163, 559 (1994).

[3] V. A. Walters, S. D. Colson, D. L. Snavely, K. B. Wiberg, and B. M. Jamison, J. Phys. Chem. 89, 3857 (1985).

[4] C. S. Parmenter and B. M. Stone, J. Chem. Phys. 84, 4710 (1986); P. J. Timbers, C. S. Parmenter, and D. B. Moss, ibid. 100, 1028 (1994).

[5] R. M. Lees, Phys. Rev. Lett. 75, 3645 (1995).

[6] M. Latka, P. Grigolini, and B. J. West, Phys. Rev. A 50, 1071 (1994).

[7] S. Tomsovic and D. Ullmo, Phys. Rev. E 50, 145 (1994).

[8] R. Roncaglia, L. Bonci, F. M. Izrailev, B. J. West, and P. Grigolini, Phys. Rev. Lett. 73, 802 (1994).

[9] R. Utermann, T. Dittrich, and P. Hänggi, Phys. Rev. E 49, 273 (1994).
[10] I. Kleiner, J. T. Hougen, R. D. Suenram, F. J. Lovas, and M. Godefroid, J. Mol. Spectrosc. 148, 38 (1991); 153, 578 (1992); S. P. Belov, M. Yu. Tretyakov, I. Kleiner, and J. T. Hougen, ibid. 160, 61 (1993).

[11] W. G. Harter and C. W. Patterson, J. Chem. Phys. 80, 4241 (1984).

[12] K. Husimi, Proc. Phys. Math. Soc. Jpn. 22, 264 (1946).

[13] C. G. Martens, J. Chem. Phys. 96, 1870 (1992).

[14] J. Ortigoso and J. T. Hougen, J. Chem. Phys. 101, 2710 (1994).

[15] W. G. Harter, Comput. Phys. Rep. 8, 319 (1988); I. M. Pavlichenkov, Phys. Rep. 226, 175 (1993).

[16] K. Takatsuka and H. Ushiyama, J. Chem. Phys. 51, 4353 (1995).

[17] E. J. Heller, J. Phys. Chem. 99, 2625 (1995). 